\title{
Laser Propagating through Air Sets Stability Record
}

\author{
An optical link for communication between distant atomic clocks is 100 \\ times more stable than previous links and could enable new precision \\ tests of general relativity.
}

By Dan Garisto

$\prod$

o look for cracks in the general theory of relativity, researchers want to compare-with unprecedented precision-the ticking of two atomic clocks, one on Earth and one in space. An ultrastable laser link will be needed for the ground-to-satellite communication, and now a team has sent a laser beam $2.4 \mathrm{~km}$ through air with its frequency stable at the level of 6 parts in $10^{21}$ for a 5-minute measurement time [1]. This result represents a 100 -fold improvement over previous efforts and demonstrates 1000 times higher stability than the best atomic clocks.

General relativity isn't compatible with quantum mechanics, and finding violations of relativity's predictions could point the

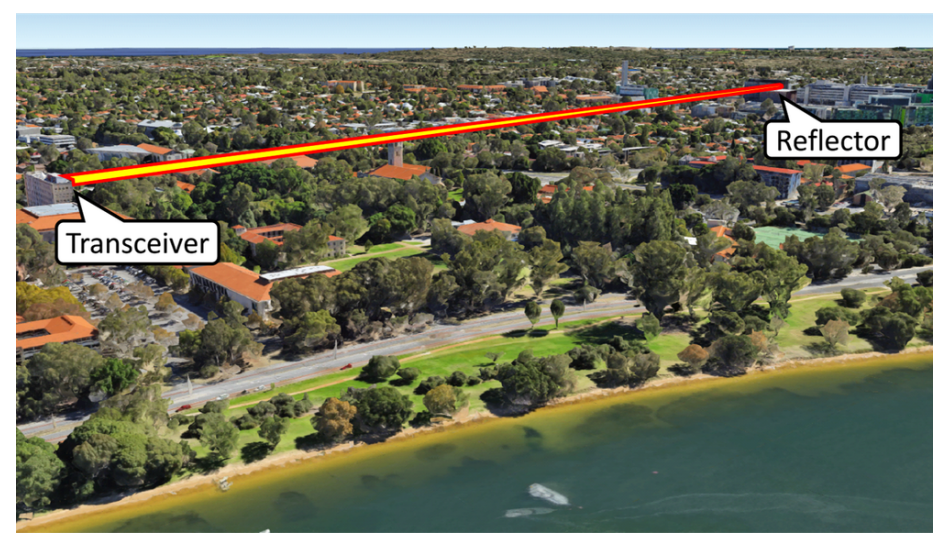

Lighting up the sky. The laser beam used to test laser link technology went on a $2.4-\mathrm{km}$ round-trip route across the UWA campus in Perth.

Credit: D. Gozzard/UWA way toward a quantum theory of gravity. According to general relativity, a clock that is higher up in a gravitational field ticks faster than one that is lower down. In 2018, researchers reported a test of this so-called gravitational redshift prediction using atomic clocks onboard two satellites with elliptical orbits. They recorded tiny frequency shifts attributable to changes in altitude, which provided a relativity test with a precision of about one part in 100,000 [2].

For higher precision, researchers would like to compare a clock on Earth with one in space. "Of course, you can't run fiber optic cable to a satellite," says David Gozzard, of the University of Western Australia (UWA), Perth. Instead, the two clocks need to be connected by a laser beam transmitted through the atmosphere.

If researchers can create a laser link stable enough for this purpose, then such links could also connect a network of atomic clocks in space that could reveal signs of dark matter or that could allow high-precision mapping of anomalies in the Earth's gravitational field. The main challenge for this technology is atmospheric turbulence-churning pockets of air cause fluctuations in laser transmission. Atomic clocks are so stable that extremely clean transmission is required to take full advantage of their precision, Gozzard says. Recent demonstrations have reduced the link instability below the level of fluctuations in the best atomic clocks-about one part in $10^{18}$-at least for certain link lengths.

In March of last year, the Boulder Atomic Clock Optical Network (BACON) Collaboration reported that their $1.5-\mathrm{km}$ laser link was stable to 5 parts in $10^{19}$ over a period of minutes [3]. Gozzard 


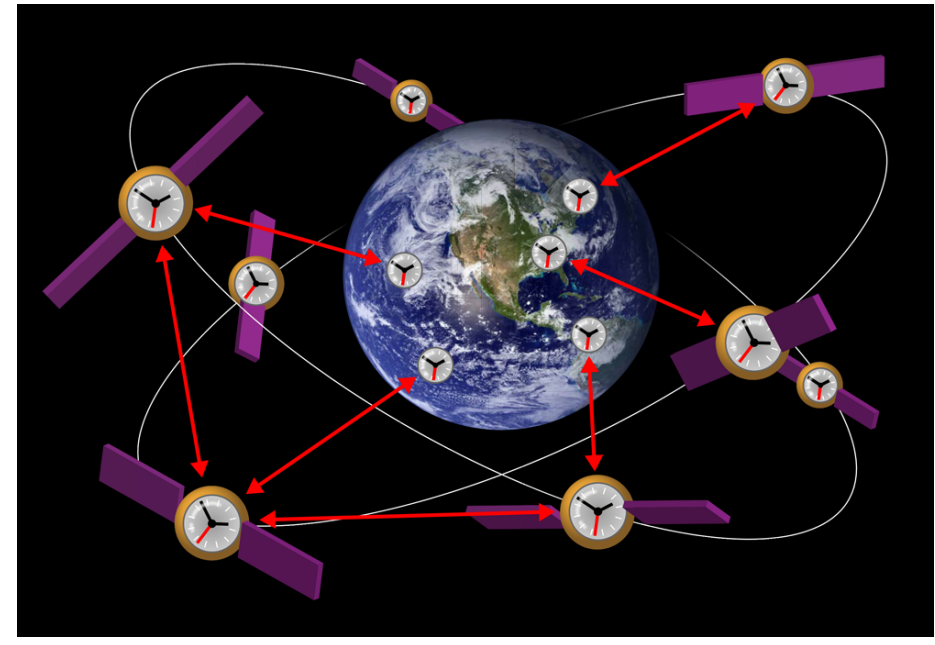

Clocking relativity. Future clock networks could use laser links (red) to synchronize atomic clocks on the ground and onboard satellites for use in dark matter searches and high-precision relativity tests. Credit: APS/Alan Stonebraker

and his colleagues have now beaten this stability by about 100 times using a different method that involves a continuous rather than a pulsed laser.

The UWA team shot a laser beam from a fifth-floor window horizontally across $1.2 \mathrm{~km}$ to a reflector, which sent it back to a receiver near the source. The turbulence experienced by the beam across $2.4 \mathrm{~km}$ was equivalent to the expected ground-to-satellite turbulence over hundreds of kilometers, Gozzard says, because air near the ground is denser and more turbulent than it is in the upper atmosphere.

To reduce intensity fluctuations, the team used a mirror in the output setup that could constantly change its tilt to compensate for slight shifts in the returning beam's location. To reduce fluctuations in the phase of the laser-which affects the frequency stability-Gozzard and his colleagues used standard noise-reduction techniques, similar to the BACON Collaboration. The UWA researchers also employed stringent temperature controls, since slight temperature variations cause voltage changes in the wires of the measuring system. Together, the noise mitigation reduced instabilities to 6 parts in $10^{21}$ for a 5-minute measurement.

Compared with pulsed lasers, continuous wave lasers send more information during a given period of time, which allows faster averaging of fluctuations and ultimately higher stability. However, Gozzard acknowledges that pulsed laser links like BACON's have some advantages, such as a superior ability to resume transmission after a dropped signal.

"Both systems beat the current best atomic clock, so we're splitting hairs here, but our ultimate precision is better," he says. Gozzard also points to the smaller size of the UWA system, which is better for packing into a satellite. To prepare for an eventual ground-to-space link, Gozzard and his colleagues are planning to test laser links over longer horizontal distances; they also expect to test ground-to-air links with light aircraft or a high-altitude balloon.

Seung-Woo Kim, an engineer at the Korea Advanced Institute of Science and Technology points out that "yet-unknown sources of degradation" could still harm transmission stability for an actual ground-to-space link hundreds of kilometers long. But he agrees that the UWA result is rigorous and suggests that, given the latest laser link results, the limiting factor may no longer be link instability. "Clock comparison between ground and low Earth orbit will be limited by the stability of the clocks themselves," he says.

Dan Garisto is a freelance science writer based in New York.

\section{REFERENCES}

1. D. R. Gozzard et al., "Ultrastable free-space laser links for a global network of optical atomic clocks," Phys. Rev. Lett. 128, 020801 (2022).

2. S. Herrmann et al., "Test of the gravitational redshift with Galileo satellites in an eccentric orbit," Phys. Rev. Lett. 121, 231102 (2018).

3. K. Beloy et al. (BACON Collaboration), "Frequency ratio measurements at 18-digit accuracy using an optical clock network," Nature 591, 564 (2021). 